\title{
Wearables as Augmentation Means: Conceptual Definition, Pathways, and Research Framework
}

\author{
Abdul Sesay \\ University of Georgia \\ Abdul.sesay@uga.edu
}

\author{
Jacob Heaton Steffen \\ University of Georgia \\ Jacob.steffen@uga.edu
}

\begin{abstract}
Wearables pervade many facets of human endeavor, thanks to their integration into everyday artifacts and activities. From fitness bands to medical patches, to augmented reality glasses, wearables have demonstrated immense potential for intelligence augmentation (IA) through human-machine symbiosis. To advance an understanding of how wearables engender IA and to provide a solid foundation for grounding $I S$ research on wearables and $I A$, this study draws from Engelbart's framework for augmenting human intellect to: (1) develop a conceptual definition of wearable technology as a digitally enhanced bodyborne device that can augment a human or non-human capability by affording context sensitivity, mobility, hands-free interaction, and constancy of operation, (2) extend Engelbart's framework to the sociomaterial domain to account for the emergence of augmented capabilities that are neither wholly social nor wholly material, and (3) propose and elaborate four augmentation pathways -complementation, supplementation, mediation, and mutual constitutionto facilitate $I A$ research.
\end{abstract}

\section{Introduction}

In a world of ubiquitous technologies where everyday experiences are lived in a cauldron of social and material intensities, the entanglement of the material and the social is no longer merely abstract, it is the reality. This is especially the case with wearables, which are increasingly popular among individual users, and are gaining momentum in organizations. Wearables are a class of digitally enhanced technology devices (e.g., glasses, watches, shoes, bands, clothes, cameras, etc.) that can be worn on almost any part of the human anatomy [32]. This includes body-worn computing devices that are integrated with electronic components, such as watches and wristbands, and smart clothing or textiles that are interwoven with sensing devices [2]. Intelligence Augmentation (IA) is the use of computers to enhance human intelligence [18]. Also referred to as Intelligence Amplification [11], IA envisages the emergence of a human-machine symbiont that "will exhibit more of what can be called intelligence than an unaided human could." While the human desire for selfimprovement through adornment can be traced back to pre-historic times, the current interest in wearables can be traced to the April 2012 unveiling of the Google 'Glass' augmented reality technology [25]. Since then, wearables have proliferated with 2014 heralded as the "Year of Wearable Technology" [12]. The wearables market is projected to grow at a compounded annual rate of $18.2 \%$, with shipments of 240 million devices in 2021 [17].

Unlike smartphones that require constant human attention for interaction, wearables permit digitallymediated experiences through hands-free operation [6, 23 ]. The growth in the number and diversity of wearable technology devices has generated awareness and interest among the public, and stimulated research and development of several applications, especially in medical care, sports and fitness, security and surveillance, big data, and the "quantified self." Wearables have the potential to transform employees and organizations into quantified dashboards, and sources or instruments of data collection to achieve organizational goals [29, 31]. A few organizations have introduced or piloted wearable technology in the workplace. In 2015, Hitachi Corporation of Japan introduced a wearable badge that tracks employee movements, job functions, and interactions with coworkers, to determine how interactions between individuals, teams, and work impact job performance [16]. UPS, a global logistics company, and Tesco, a UK retail company, have used wearable technology to improve employee productivity in warehouse operations [35]. And recently in the US and elsewhere, police organizations have implemented body-worn cameras to document evidence of police-civilian interactions, and thereby increase transparency and accountability of police operations [15]. 
Wearables have immense potential to augment human capabilities through perceptive, cognitive as well as physical means [2, 6, 11, 22, 23, 24]. For example, wearables can be leveraged to eliminate managerial risk, elevate performance, and extend employee competencies and capabilities [32] by seamlessly integrating on-demand information with everyday tasks. Wearables are projected to play a central role in the development and realization of the Internet of Things (IoT), by mediating how people interact with so-called smart objects-tiny devices equipped with a microcontroller, wired or wireless communication interface, power supply, sensors and actuators that are used to interface with the surrounding environmentusing voice, gestures, or touch controls [7]. In recent years, platform convergence has availed various artificial intelligence (AI) methods [13], such as machine learning (ML), Virtual Reality (VR), Natural language processing and speech recognition, to common business problems. To a large extent, the success of these methods depends on the proliferation of Big Data, which is increasingly being collected and harnessed by smart objects embedded in wearables [7, 26]. Thus, wearables are feed and fodder for the IoT, and a point of convergence for AI and IA.

Although wearables are popular and show immense potential to augment human capabilities through human-machine symbiosis, the concept is still undertheorized, and offers significant opportunities for research. First, to the best of our knowledge, no conceptual definition exists to tie all the capabilities of wearables together into a unified framework for research. As the editors of a recent special issue of ISR note: "there is still a lack of coherent discussion and an integrated body of literature on the direct implications of how IA and AI research can contribute to organizational and societal applications and to their impact on the future of work" [18]. Second, extant studies on wearables have treated them as artifacts [20] that exist separate and apart from the humans who wear them. Thus, how wearables relate to humans, and vice versa, has not been explored. Last, but not the least, we lack an understanding of how wearables can be incorporated and harnessed in a framework to engender IA or human-machine symbiosis.

Thus, given the novelty of wearables and their potential to augment human capabilities in individual and organizational contexts, we are motivated to ask: (1) From a sociomaterial (relational) perspective, what makes a technology wearable, and (2) how does wearable technology engender intelligence augmentation? By addressing these question, we hope to contribute to the literature on IA and sociomateriality by (1) developing a conceptual definition of a wearable as a digitally enhanced body-borne device that can an augment a human or non-human capability by affording context sensitivity, mobility, hands-free, interaction, and constancy of operation, (2) extending Engelbart's two-domain human augmentation framework by incorporating a sociomaterial domain to account for the emergence of new capabilities that are neither wholly human nor wholly material, and (3) elaborating four pathways - complementation, mediation, supplementation, and mutual constitution-that explicate the various means of human augmentation.

The rest of this paper is organized as follows. First, we review the literature on wearables and Engelbart's augmentation framework, followed by the development of a conceptual definition and augmentation means and pathways. We then provide a framework for intelligence augmentation and discuss its amenability for research in wearables and human-machine symbiosis.

\section{Literature Review}

As a recent phenomenon, academic studies examining wearables from an IS theoretic and organizational perspective are few. James et al. [20] categorized wearable technology features into three sets (social interaction features, exercise control features, and data management features) and investigated how the use of each feature set is influenced by individuals' exercise motivations. Drawing from self-determination theory and affordances, they concluded that individuals "with different motivations toward exercise have unique fitness technology use profiles," and that "to achieve the most effective outcomes ... it may be wise to consider personalizing use to the users' characteristics." These findings imply that when it comes to personalized wearable technologies, a reductionist one-size-fits all approach may not be appropriate. Rather, how each user relates to the technology and the motivations for such relations should be considered. Prasopoulou [29] provided a first-person account of the relationship between a wearable technology (Fitness Tracker), and the wearer by recounting how recording "both the mundane, repetitive actions and the extraordinary moments of life with wearables" allowed her to "capture the raw experience of humans in a systematic effort to analyze their encounters with digital devices and data in the Internet of Things". While referencing experiential computing [40], the study calls for a shift in current sociomaterial research "in the form of algorithms [27], robots [4] and computer grids to a focus of the body. Within mainstream IS conference proceedings and other allied publications, preliminary studies on wearables are beginning to appear. Benbunan-Fich [5] used an affordance framework to investigate whether the absence of visible interaction cues in minimalist wearable devices, such as the Fitbit Flex, affects the user 
experience. Describing minimalist as the absence of visible interaction cues, the study concluded that minimalist designs engender complex user experiences due to data inaccuracies and inconsistences and contradictions in integrating the devices with the web platform. However, motivational effects (such as feedback and goal setting with teams) may outweigh minimalist design concerns among certain users. This observation aligns with the conclusion by James et al. [20] that users' profiles of fitness technology feature set use correspond with the motivation to exercise.

Notwithstanding their enormous potential to revolutionize fitness and healthcare, preliminary studies on the adoption and efficacy of wearables have produced mixed results, not to mention the commercial failure of Google Glass, the harbinger of the current craze on wearables. For example, in a randomized clinical trial to test the efficacy of wearable technology as a weight loss intervention, Jakicic et al. [19] concluded that "devices that monitor and provide feedback on physical activity [wearables] may not offer an advantage over standard behavioral weight loss approaches". Similarly, James et al. [20] found that "social interaction and data management features of current fitness technologies show promise in assisting well-being outcomes, but only for the more selfdetermined and amotivated subtypes of exercisers". Furthermore, a 2014 commercial study by Endeavour Partners showed that $30 \%$ of users of activity trackers stop using their wearable device within six months of acquiring them (cited in [8]). We believe that a confounding factor in these studies is the lack of conceptual clarity about wearables. This is evident in the many definitions of wearables adopted by researchers [6]. While this is acceptable for general discourse, it poses challenges for developing and accumulating a deeper understanding of the relationships between wearers and wearables.

These studies have evidently contributed to our nascent understanding of wearables. However, questions about the types of relationship between wearables and wearers have not been explored. This ignores a salient feature of wearable technologyintegration into the performance of everyday activities. As Yoo [40] observed: "digitalized artifacts play decisive roles in shaping and mediating all dimensions of our lived experiences. Yet, there is a serious intellectual void that needs to be filled to understand exactly the nature and the consequences of the digital mediation of human experiences." Similarly, Prasopoulou [29] urges IS research to "focus on the body (flesh, feelings, and thoughts) for sourcing knowledge on human-non-human encounters in emerging cyber-physical spaces like the Internet of Things." And James et al. [20] suggested the need to extend traditional motivational theories "to accommodate advances in personal informatics technologies that allow users to customize the environment in which they are performing activities."

Given that wearables can instantiate human-machine symbiosis, it is crucially important to build a solid foundation on which to ground a broader understanding and conceptualization of these relationships, beyond the mere fact that the technology is worn on some part of the human anatomy. This is what motivates us to find out what makes a technology wearable, and how does it engender IA. Wearables present an interesting case of the intimate relationship between humans and technology at a conceptual and literal level, involving sensory, mental, and motor capabilities [24, 11]. For example, wearables that are ingested (e.g., the Abilify MyCite pill) or implanted [24] in the body, exemplify an inseparably entangled relationship between the wearable technology and the wearer. On the other hand, a wrist band, such as Fitbit or Apple Watch that can be worn and taken off at ease, exemplifies a different level of entanglement, where the conceptual relationship may be different from the literal one.

\section{Wearables and Human Augmentation}

Licklider [21] pioneered the concept of human-machine symbiosis to unleash the power of machines from performance of mundane tasks involving preformulated or predetermined computation, to partners in the "formulative part of technical problems" and in the "process of thinking that must go on in "real time"". As he puts it: "If those problems can be solved in such a way as to create a symbiotic relation between a [hu]man and a fast information-retrieval and data-processing machine ... it seems evident that the cooperative interaction would greatly improve the thinking process". Thus, human-machine symbiosis portends a shift from a deterministic human-machine relationship to a synergistic relational one, based on the thinking process. While Licklider laid the intellectual foundation for the ascension of computing machines as "thinking" partners in human-machine symbiosis, Engelbart [11], inventor of the mouse, developed the framework for human augmentation. In his seminal report on "Augmenting Human Intellect," Engelbart [11] employed a systems approach to develop a conceptual framework, which "can include many things - all of which appear to be but extensions of means developed and used in the past to help man apply his native sensory, mental, and motor capabilities." According to Engelbart's framework, the quickest route to augmentation involved two paths: (1) access to minuteby-minute computer services, and (2) methods of thinking and working to leverage the power of the 
computer. These two paths delineate the mode and means of augmentation, and embody the axiomatic definition of wearables as computerized or digitallyenhanced devices. The computerization requirement eliminates devices such as eye glasses, wristwatches and ordinary clothes from consideration in Engelbart's augmentation framework. Since wearables are expected to provide continuous, on-going computer services to the human wearer (mode), and also afford quantification and analysis of data to derive and leverage information from use (means), they can provide a solid foundation for investigating IA.

Wearables increasingly operate to blur the boundaries between human and non-human performativity, ushering non-trivial improvements in specific tasks and performance outcomes. Thanks to the digitalization and miniaturization of computer and communication devices, wearables are now integrated into everyday artifacts and activities, and have become pervasive in many facets of human endeavor. For many contemporary challenges and opportunities in healthcare and wellness, fitness and entertainment, surveillance and monitoring, wearable technology has become indispensable. Examples include, fitness bands to promote physical activity for a healthy lifestyle, medical patches that track ingestion of life-saving drugs, augmented reality glasses that download reams of information to the pupil of the eye, and wearable cameras that provide raw, unfiltered, and objective audio-visual information from a first-person perspective. Three principles make wearables transcend the status of simple tools, making them suitable for IA: mobility/constancy of operation, context sensitivity, and augmentation $[6,23]$. It is worth clarifying that augmentation as a characteristic of wearable technology is not equivalent to IA. Augmentation is a capacity that may or may not be exercised during human-machine symbiosis, whereas IA is an emergent outcome of human-machine symbiosis [11, 21]. IA can be achieved through any means of human-machine symbiosis (e.g. algorithmic decision-making) that does not necessarily involve wearables. Thus, in this study, we refer to augmentation only in the narrow sense of wearable augmentation, and IA in the broader sense of an emergent sociomaterial outcome that may or not involve wearables.

\section{Mobility and constancy}

Mobility and constancy of operation embody the vision of ubiquitous computing, where computers become woven "into the fabric of everyday life until they are indistinguishable from it" [39]. It exemplifies Engelbart's mode of augmenting human intellect with on-going computer services. When computers become part of our wardrobe, "our computer system will share our first-person perspective and will begin to take on the role of an independent processor, much like a second brain - or a portable assistant ... As it 'sees' the world from our perspective, the system will learn from us, even when we are not consciously using it" [22]. Mobility and constancy of operation is made possible by the availability of an external power source, such as a battery or LED. This is a key distinguishing feature between classes of wearables that are programmable and those that are not, as is the case with the simple prosthetic and the bionic leg. In addition to mobility and constancy of operation, wearables are designed to afford hands-free operation, much unlike other mobile devices, such as cell phones and laptops. This allows the wearer to integrate virtual information into their personal domain, without detaching themselves from the physical world around them.

\section{Context sensitivity}

A wearable computer has context sensitivity to the wearer's physical environment and physiological state, which can be exploited to provide appropriate responses to environmental stimuli [6] or manipulate the wearer's emotional state to do something [28]. Context sensitivity of wearables falls into two broad categories - situational awareness and situational unawareness. Wearables that can provide information about the wearer and the world around them are said to be situationally-aware, and those that provide information relevant to the task at hand but are not computationally aware of their surrounding are situationally-unaware [14]. For a wearable to be situationally-aware, it must have capabilities for identification, processing, communication, and storage (IPCS). These capabilities allow the wearable to be discoverable in a network, and to maintain a stored (past), current (present), and predictive (future) state [3]. A situationally-aware wearable can be triggered by environmental or physiological stimuli, such as an activity, policy, or process [26], and respond autonomously as needed. For example, most fitness trackers and smartwatches are able to record fitness data and directly connect to the Internet and communicate with web services. Situational awareness, perceptual intelligence, and a first-person perspective makes it possible for wearables to augment human capabilities and assist with day-to-day activities [22].

Context sensitivity allows wearables to "sense" various kinds of environmental stimuli that, depending on specific applications, make it possible for appropriate interception and mediation of signals. For example, using a geographic positioning system (GPS) application, a wearable can allow the interception and processing of location signals. These signals can then be used to provide location-specific information to the 
wearer (for example, giving them directions from a particular location to another location). Additionally, wearables designed to make bodily contact with the wearer can sense and process physiological signals, which can trigger a specific or programmed response. As the wearer of a fitness tracker attests: "The tracker carried this great promise of unlocking information on one's own body. It would be a lie to say that I was not influenced by it. So much so, that I systematically followed the suggested use: wearing it all the time, and monitoring my steps [29; emphasis added]. In medical applications, situationally-aware wearables have been used to monitor vital signs, such as heart rate, blood pressure, and blood glucose levels of the wearer, and alert them or a caregiver to take appropriate actions.

Situationally-unaware wearables could have potential information that could be made available to the Web, but do not have the necessary capabilities to communicate over TCP/IP or HTTP. However, if they are uniquely identifiable, they could be afforded additional resources to communicate [26]. This is the case with the current class of police body-worn cameras, which lack the native capability to directly communicate to the Internet. However, they are uniquely identified, and are provided with docking stations which allow them access to connect and communicate to the Internet.

\section{Augmentation}

Much of the early research on wearable technology, principally undertaken by computer scientists and engineers, was preoccupied with how to use wearables to augment human capabilities [36, 14, 6, 23, 11]. An example is the Remembrance Agent, which was designed to enhance memory through "intelligent filtering and proactive presentation" [6]. The foundational work for the use of computing devices to augment human capabilities was laid by the eminent computer scientist, Doug Engelbart. In his seminal report: "Augmenting Human Intellect: A Conceptual Framework," [11] defined "augmenting human intellect" to mean: "increasing the capability of a man to approach a complex problem situation, to gain comprehension to suit his particular needs, and to derive solutions to problems". Increased capability in this respect is taken to mean a mixture of the following: more-rapid comprehension, better comprehension, the possibility of gaining a useful degree of comprehension in a situation that previously was too complex, speedier solutions, better solutions, and the possibility of finding solutions to problems that before seemed insoluble." For a wearable technology to augment a human capability, it must interact with the wearer either by mediating between the wearer and the world [2], or by becoming a constitutive and inseparable part of the wearer. For example, a fitness tracker interacts with a wearer by mediating between the wearer and a specific task, say exercise. In this case, the wearable (fitness tracker) does not actually perform the task; it mediates through extrinsic motivations [20] to get the wearer to do something (exercise). On the other hand, the Abilify MyCite ingestible pill and patch system is constitutive with the wearer. Once ingested, the pill and patch become part of the wearer and provide therapeutic remedy without further intervention by the wearer. In each case (mediation or constitutive), the wearable provides a means to manipulate information flows from the world and the wearer. The information flows may directly alter the wearer's sensory, mental, or motor capabilities, or allow the wearer to edit, store, or otherwise act on the information [28, 2].

Wearables can be designed to augment human perception and cognitive capabilities by mimicking the five major senses-vision, audition, olfaction, touch, and taste). They can do so by: (1) restoring or compensating for a lost or diminished human capability to bring it within "normal" range of operation. For example, a bionic leg can restore a diminished human capability to walk on two legs, (2) increasing the range of the capability, and (3) adding a new capability

\section{An Augmentation-Based Definition of Wearable Technology}

Given that people wear technology not only for the sake of adornment or sartorial vanity, we propose a definition of wearable technology that pays homage to both form and function. Based on the overall conceptual development and exposition of wearables in the IS and computer science domains, we define a wearable technology as a digitally enhanced body-borne device that can augment a human or non-human capability by affording context sensitivity, mobility, hands-free interaction, and constancy of operation.

\section{Means and pathways to IA}

The proposed definition satisfies all the elements of wearable technology - mobility/hands-free/constancy of operation, context sensitivity, and augmentation. A major difference between the proposed definition and extant ones is the focus on form and function, rather than just form or identity. Essentially, the proposed definition addresses the question of what makes (or how is) a technology wearable. It starts from the axiomatic assumption that technology is a means to an end. In this case, augmentation is the means and an augmented or improved human or non-human capability is the end [11]. The notion of capability allows us to acknowledge the fact that augmentation of the human can proceed via non-human means, such as artificial intelligence (AI), 
machine learning (ML), Virtual Reality (VR), etc., and thereby extend Engelbart's framework from a twodomain (human/social and artifact/material) system to a three-domain (social, material, and sociomaterial) system.

\section{Augmentation Means}

Engelbart [11] defined four basic classes or augmentation means by which human capabilities can be extended:

(1) Artifacts - physical objects designed to provide for human comfort for the manipulation of things or materials and for the manipulation of symbols. In this study, the focal artifact is a wearable technology, and its context of use is in the sociomaterial domain. This is because, in concept and by design, a wearable operates as part and parcel of its human host. Both the human (social) and the technology are needed to constitute intelligence augmentation.

(2) Language - "the way in which the individual parses out the picture of his world into the concepts that his mind uses to model that world and the symbols that he attaches to those concepts and uses in consciously manipulating the concepts ("thinking")." Here, language includes both human language (declarative as well as performative utterances), and symbolic computer programming languages, such as $\mathrm{C} / \mathrm{C}++$, Java, etc. Through language (human and machine), dialog between the wearer and the wearable provides a means for information exchange and synergistic processing. Language is conceptualized as a selforganizing system that can affect "its own evolution to a succeeding state" [11].

(3) "Methodology - the methods procedures strategies, etc., with which an individual organizes his goal-centered (problem-solving) activity." Methods provide the means to constitute and manipulate humanmachine symbiosis. Advances in speech recognition, AI and Machine learning techniques, provide demonstrable means to augment intelligence through Big Data analytics and goal-based instructions.
(4) Training - the conditioning and adaptation needed by the human being and the technology to bring "skills in using Means 1, 2, and 3 to the point where they are operationally effective." The augmented system can thus be visualized as emerging from the interrelationships among language, artifacts and methodology through training. Although it may be characterized that way, Engelbart's conceptualization of IA is neither deterministic nor prescriptive. It is an inclusive process for "a way of life in an integrated [human-machine] domain where hunches, cut-and-try, intangibles, and the human 'feel for situation' usefully coexist with powerful concepts, streamlined terminology and notation, sophisticated methods and high-powered electronic aids". Although Engelbart did not specifically speak of wearables, his framework for providing humans with minute-by-minute access to electronic aids, is generally considered to presage the era of wearables [2]. Thus, wearables instantiate human-machine symbiosis by means of language, methodology and training, with IA as the desired outcome. The strong interrelationship among these augmentation means can account for any potential changes in IA processes or pathways [11].

\section{Augmentation Pathways}

Whereas augmentation means define the resources or antecedents necessary for the realization of IA, augmentation pathways define the methods which guide the means to IA. As discussed above, human perception and cognitive capabilities can be augmented to restore a diminished capability to an acceptable or normal range; increase an existing capability; or add a new capability [2]. Each of these possibilities represent a pathway to IA (see Table 1). We propose four pathways, which are derived from unique configurations of the basic characteristics of wearables that match a particular range of augmentation.

\begin{tabular}{|l|l|l|l|l|}
\hline \multicolumn{5}{|c|}{ Table 1. Augmentation Pathways for Wearable Technology } \\
\hline Pathways & Complementation & Mediation & Supplementation & Constitution \\
\hline Augmentation & Within range & Increase range & Add range & Add range \\
\hline Context Sensitivity & Unaware & Aware & Unaware & Aware \\
\hline Constancy & Not powered & Powered & Not Powered & Powered \\
\hline Example & Prosthetic leg & Bionic leg & Wing suit & Space suit \\
\hline
\end{tabular}

Complementation: According to Webster's New Universal Unabridged Dictionary, to complement is "to provide something felt to be lacking or needed; it is often applied to putting together two things, each of which supplies what is lacking in the other, to make a complete whole." Thus, in complementation, the wearable adds something needed by the human to compensate for something missing or lacking. The 
augmentation is done to bring the lacking capability to "within normal range" of human capability. For example, consider the case of a soldier who loses a limb in battle. The loss of the leg diminishes the soldier's capability to walk on two legs, as is customary for ablebodied human beings. In order to restore the soldier's ability to walk, a simple prosthetic leg (a wearable technology) may be worn by the soldier as needed. The prosthetic leg may or may not be computerized, and can function with or without situational awareness. Thus, complementation provides a pathway to augmentation that matches the configuration of context sensitivity and constancy of operation depicted in Table 1. We now formally define "Complementation" as: a pathway to IA that uses a wearable technology to compensate for, restore, or augment a diminished/compromised human capability.

Mediation: Mediation generally means "acting through, dependent on, or involving an intermediate agency" to effect an agreement or designate processes for bringing an agreement or reconciliation. Of note is that the solution brought about by a mediation is not binding or mandatory by the parties involved (Webster's New Universal Unabridged Dictionary 1996). In the case of wearables, mediation often involves a computerized system that intermediates between human (social) and technology (material) inputs. In mediation, the wearable technology, which is often computerized, does not lead directly to the desired outcome or augmented capacity. Rather, it receives and processes information signals from the wearer (be they physiological, cognitive, or physical), and provides feedback that the wearer then uses to augment a capability. Take the case of a health-conscious user of a popular fitness tracker. The user's goal may be to improve cardiovascular fitness by running 10 miles per week. Evidently, the fitness tracker will not do any running for the wearer. In fact, the runner can choose to run with or without the tracker and still meet the fitness goals. However, by providing alerts to the runner, and by keeping track of the runner's progress, and by providing feedback regarding progress towards goals, the runner may make cognitive decisions in consonance with the tracker's feedback and suggestions. Again, it is worth emphasizing that this relationship is neither mandatory nor deterministic. The achievement of the fitness goal is conceivable without a fitness tracker. But with a fitness tracker, the goal is aided through mediation. As Table 1 shows, mediation provides a pathway to augmentation that matches the configuration of situational awareness for context sensitivity and constancy of operation. Mediation involves the interception and processing of signals from one entity to another and involves the intervention of sensors and computing devices. Formally, we define "Mediation" as: a pathway to IA in which a wearable technology is used to mediate between a goal/task and the performance of the task for the purpose of augmenting a human capability.

Supplementation: To supplement is to add to a person or thing. The adding is done not necessarily to provide something felt to be lacking or needed; it is simply to add to what is already available. Consider Wingsuit fliers, who jump from BASE cliffs at altitudes of over 30,000 feet, gliding for over five minutes at speeds of over 150 miles per hour, to experience the joy of flying or gliding. Certainly, gliding is not an innate human capability. Therefore, the goal is not to complement the loss or diminution of a "normal" human capability. Rather, it is to add an experience beyond what people normally do. Evidently, without the wingsuit, no human can glide. In this case the imbrication of the human (glider) and the wearable technology (wingsuit), supplements or adds a nonhuman capability-gliding. Without the wearable wingsuit, the human will not be able to glide. Thus, we formally define "Supplementation" as "a pathway to IA that uses a wearable technology to augment a nonhuman capability. In supplementation, there is a direct or mandatory fusing of the wearer and wearable in order for the augmentation means to activate. This may or may not involve the use of sensors or computing devices. For example, a wing suit is manually operable and does not require the use of sensing or computing technology. However, a space suite, which supplements a human capability to live in the adverse conditions of outer space, uses several sensors and computing devices to mediate the relationship between the astronaut and the space suit.

Constitution: To constitute is to make a thing what it is; an equal and essential part of a whole (The American Heritage College Dictionary, Third Edition). In the case of wearables, constitution involves a reciprocal supposition in which dissimilar or heterogeneous entities (social and material), though different, and each capable of independent existence, become constitutive as their thinking processes (human brain and computer processor) merge into a whole that exceeds the sum of its parts [28]. Once constituted, the components remain inseparable [34]. As Picard [28] notes: "cognitive thought involves the brain, which is a part of the body." The process of thinking, be it human, or machine, "involves biochemical and electrical signaling mechanisms: physical changes in the body." This is generally the case with implants, such as the eye tracker [23], or ingestibles, such as the Abilify MyCite system. Approved by the U.S. Food and Drug Administration in 2017, the Abilify MyCite system is comprised of a patch that can be attached on the body, and a pill coated with a digestible chip. When ingested, 
the pill sends signals to the patch worn by the patient. The signals can be processed with a phone or Web App to determine appropriate care for the patient. Constitution provides a pathway to augmentation that matches the configuration of situational awareness for context sensitivity and constancy of operation. However, unlike mediation, a new capability is added as a result of the augmentation (see Table 1). Formally, we define "constitution" as: a pathway to IA in which a wearer and wearable technology become inseparably entangled to augment a human or non-human capability.

The above exposition delineates the various means and pathways linking the relationship between a wearer and a wearable technology toward IA. It validates "the picture of dissimilarity" [21] between the human and the machine, while acknowledging the emergent outcome (augmentation) of their coming together. The pathways run the gamut from seemingly separable complementation encounters to inseparable constitutive entanglements. As Licklider [21] observes: "It seems likely that the contributions of human operators and equipment will blend together so completely in many operations that it will be difficult to separate them neatly in analysis. ... In other operations, however, the contributions of men and equipment will be to some extent separable."

\section{Augmentation framework}

Figure 1 illustrates a conceptual model of IA that incorporates the means and pathways articulated above. The framework consists of three major components, each numerically labelled with a dark circle: (1) Means, (2) Pathways, and (3) Outcome-Intelligence Augmentation. The components are connected by paths $a$, through $f$, representing the links between means, pathways, and outcome. The combination of means in component 1 with any of the pathways in Component 2 can engender IA in component 3. Complementation and Supplementation pathways can directly engender IA without the situational awareness provided by sensing mechanisms, and the constancy of operation provided by a power source, such as a battery or LED. Typically, the non-powered pathways to IA do not meet Engelbart's requirement for access to on-going computer services, nor do they have the "thinking" capability envisaged in Licklider's human-machine symbiosis.

Consequently,

Complementation

or Supplementation without the use of computing services is of little interest to wearable computing. In order to remedy the situation, Complementation and Supplementation pathways can be configured to go through either Mediation or Constitution, as depicted by paths $b, d$, and $c$, $e$, respectively. Mediation and
Constitution pathways must necessarily have a power source to provide constancy of operation, and a sensor to provide reciprocal feedback between the wearer and the wearable.

From figure 1 , it is clear that wearables provide effective means to IA, especially when they are configured with context sensitivity and constancy of operation. Augmentation can proceed via four pathways, complementation, supplementation, mediation, and constitution, depending on the desired range or goal. When the goal is to compensate for a diminished human capability to bring it to within normal range, augmentation can proceed via complementation and mediation. When the aim is to add a new capability, augmentation can proceed via supplementation or constitutive pathways.

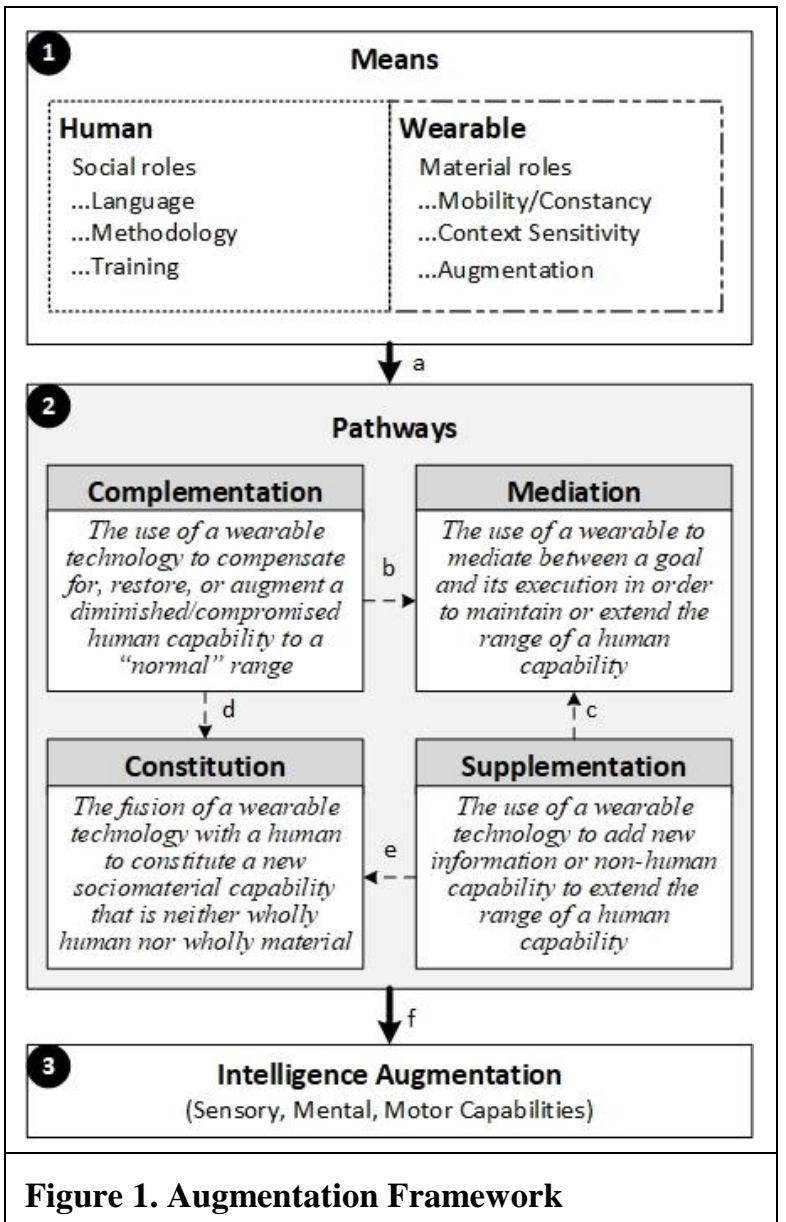

With advances in ingestible and implantables, wearables portend a future where the most profound uses will be constitutive. This will mean a one-size-fitsall approach will be less effective [20]. Rather, based on notions of the quantified self $[38,33]$, wearables will be tailor-made to the constitution of a specific individual, or group of individuals. This will facilitate the self- 
tracking of biological, physical, behavioral, or environmental information that can be acted upon by the individual [38]. Such a future is contemplated by the French philosophers, Deleuze and Guattari [9], when they write about the heterogeneity of assemblages as: "a pure multiplicity of immanence, one piece of which may be Chinese, another American, another medieval, another petty perverse, but all in a movement of generalized deterritorialization in which each person takes and makes what she or he can, according to the tastes she or he will have succeeded in abstracting from a Self [Moi]."

\section{Discussion and Conclusion}

As information systems have become ubiquitous, and the Internet of Things gradually becoming a reality, there is a growing need to leverage wearables as the nexus between things and humans. More than a half century ago, when Licklider [21] and Engelbart [11] laid the foundation for human-machine symbiosis and intelligence augmentation, advances in computer hardware and software have progressed at breathtaking speeds, inching ever closer to the reality of "thinking" machines that simultaneously complement and augment human intellect. However, this vision may be delayed or scuttled by the lack of conceptual clarity about wearables, as evidenced by the many definitions that abound. In this regard, we posed two research questions:

1) From a sociomaterial (relational) perspective, what makes a technology wearable?

2) How does wearable technology engender intelligence augmentation?

We addressed the first question by developing an augmentation-based definition of a wearable as $a$ digitally enhanced body-borne device that can an augment a human or non-human capability by affording context sensitivity, mobility, hands-free interaction, and constancy of operation. We argue that this definition focuses on form and function and satisfies all the elements of wearable technology - mobility/handsfree/constancy of operation, context sensitivity, and augmentation. Additionally, the definition focuses on capabilities, which allows us to acknowledge the fact that augmentation of the human can proceed via nonhuman means, such as artificial intelligence (AI), machine learning (ML), Virtual Reality (VR), etc., and thereby extend Engelbart's framework from a twodomain (human/social and artifact/material) system to a three-domain (social, material, and sociomaterial) system.

To address the second question, we appropriated and extended the framework for augmenting human intellect [11]. We argue that the strong interrelationships among language, method and training can account for any potential changes in IA processes for any sociomaterial domain (e.g., human and wearable). To guide augmentation means to a desired outcome, we elaborate four pathways that can directly or indirectly channel augmentation means-complementation, mediation, supplementation, and constitution. These pathways can be employed based on the desired range or goal of augmentation. When the goal is to compensate for a diminished human capability to bring it to within normal range, or increase its range, augmentation can proceed via complementation and mediation. When the aim is to add a new capability, augmentation can proceed via supplementation or constitutive pathways. These pathways have significant implications in the notion of a quantified self.

Our conceptual definition of wearable technology contributes to theory by ensuring consistency and attaining a high level of coherence among the widely recognized elements of wearables. As Suddaby [37] points out, a good conceptual definition must "effectively capture the essential properties and characteristics of the concept." By articulating the various configurations of constancy of operation, context sensitivity, and augmentation, we exhausted the key identifying characteristics of wearables and provided a definition that will be consistent across all manner and types of wearables. Additionally, rather than focusing on problematizing wearables per se, we abstracted to the archetypal concept of augmentation, to safeguard against what Rai [30] described as "Type III errors - that is, formulating a research problem so that the answer to the question will matter ... while overlooking how the problem relates to a more generic, archetypal problem." Thus, we developed an augmentation-based definition that addresses the general problem of intelligence augmentation.

Our research framework extends Engelbart's twodomain human augmentation framework by addressing intelligence augmentation as a sociomaterial problem to account for the emergence of new capabilities that are neither wholly human nor wholly material. Finally, by incorporating augmentation means and pathways, the framework provides a roadmap for researchers to develop empirical questions about IA. 


\section{References}

[1] Abilify MyCity. 2017. Https://www.abilifymycite.com Accessed on: June 11, 2019.

[2] Baber, C. 2001. "Wearable Computers: A Human Factors Review", International Journal of Human-Computer Interaction (13:2), pp. 123-145.

[3] Baber, C., Haniff, D.J., Woolley, S.I. 1999. "Contrasting Paradigms for the Development of Wearable Computers," IBM Systems Journal (38:4), pp. 551-565.

[4] Beane, M., Orlikowski, W.J. 2015. "What Difference Does a Robot Make? The Material Enactment of Distributed Coordination," Organization Science (26:6), pp.1553-1573.

[5] Benbunan-Fich, R. 2017. "Usability of Wearables without Affordances," in Proceedings of the 23rd. Americas Conference on Information Systems, Boston, MA. August 2017.

[6] Billinghurst, M., Starner, T. 1999. "Wearable Devices: New Ways to Manage Information," Computer (32:1), pp. 57-64.

[7] Cirani, S., Picone, M. 2015. "Wearable Computing for the Internet of Things" IT Pro, September/October 2015, pp. 41.

[8] Coorevits, L., Coenen, T. 2016. "The Rise and Fall of Wearable Fitness Trackers," in Proceedings of the 76th Academy of Management Conference, Anaheim, CA. August 2016.

[9] Deleuze, G., and Guattari, F. 1987. A Thousand Plateaus: Capitalism \& Schizophrenia. Trans. Brian Massumi. Minneapolis, MN: University of Minnesota Press.

[10] Deng, X.F., Christodoulidou, N. 2015. "Understanding User Values of Wearable Computing." in Proceedings of the Thirty Sixth International Conference on Information Systems, Fort Worth.

[11] Engelbart, D.C. 1962. "Augmenting Human Intellect: A Conceptual Framework", Summary Report Prepared for Director of Information Sciences, Airforce Office of Scientific Research, Washington DC., Stanford Research Institute, Menlo Park, California, pp. 1-134.

[12] Forbes Magazine 2013 accessed on April 5, 2018, accessed at: https://www.forbes.com/sites/ewanspence/2013/11/02/2014will-be-the-year-of-wearable-technology/\#5456551ac466.

[13] Grudin, J. 2009. "AI and HCI: Two Fields Divided by a Common Focus," AI Magazine, pp. 48-57.

[14] Haniff, D.J., and Baber, C. 1998. "Wearable Computers for the Fire Service and Police Force: Technological and Human Factors", in Digest of Papers of the 3rd International Symposium on Wearable Computers, Los Alamitos, CA: IEEE Computer Society, pp. 185-186.

[15] Harvard Law Review, Chapter Four: Considering Police Body Worn Cameras. April 10, 2015. (128:6), pp. 1794-1861.

[16] Hitachi High-Technologies has Developed a New Wearable Sensor the Measures 'Organization Activation Level' Correlated with Collective Happiness' Press Release, Tokyo, Japan, February 9, 2015.

[17] IDC Media Center. 2017. "Worldwide Wearables Market" accessed at: http://www.idc.com/getdoc.jsp?containerId=pr US42818517, accessed on 4/5/18

[18] Jain H., Padmanabhan, B., Pavlou, P.A., Santanam, R.T. 2018. "Special Issue of Information Systems Research-Humans, Algorithms, and Augmented Intelligence: The Future of Work, Organizations, and Society," ISR (29:1), pp. 250-251.

[19] Jakicic et al. 2016. "Effect of Wearable Technology Combined with a Lifestyle Intervention on Long-Term Weight Loss: The IDEA Randomized Clinical Trial," JAMA (316:11), pp. 11611171. doi: 10.1001/jama.2016.12858.
[20] James, T.L., Wallace, L., Deane, J.K. 2019. "Using Organismic Integration Theory to Explore the Association between Users' Exercise Motivations and Fitness Technology Feature Set Use," MIS Quarterly (43:1), pp. 287-312.

[21] Licklider, J.C.R. 1960. "Man-Computer Symbiosis," IRE Transactions on Human Factors in Electronics, pp. 4-11.

[22] Mann, S. 1997. "Cybersquare: MIT Media Lab," Computer, pp. 25-31.

[23] Mann, S. 1998. "Wearable Computing and Means of Personal Empowerment," in Proceedings of the 3rd International Conference on Wearable Computing (ICWC) pp. 51-59.

[24] Mann, S. 2014. "Wearable Computing," in Soegaard, M., Dam, R.F. (eds.). The Encyclopedia of Human-Computer Interaction, $2^{\text {nd }}$ Ed. Accessed: 4/6/18, Accessed at: https://www.interactiondesign.org/encyclopedia/wearable_computing.html

[25] MarketLine Case Study. 2014. "Wearable Technology: The New Tech Battleground?" Accessed at www.marketline.com

[26] Mathew, S.S., Atif, Y., Sheng, Q.Z., Maamar, Z. 2013." The Web of Things - Challenges and Enabling Technologies," in Internet of Things and Inter-Cooperative Technologies for Collective Intelligence, Bessis et al. Eds., Springer.

[27] Orlikowski, W.J., Scott, S. 2015. "The Algorithm and the Crowd: Considering the Materiality of Service Innovation," MIS Quarterly (39:1), pp. 201-216.

[28] Picard, R.W. 2003. "Affective Computing: Challenges," Int. J. Human-Computer Studies (59), pp. 55-64.

[29] Prasopoulou, E. 2017. "A Half-moon on my Skin: A Memoir on Life with an Activity Tracker," European Journal of Information Systems (26:3), pp. 287-297.

[30] Rai, A. 2017. "Editor's Comments: Seeing the Forest for the Trees," MIS Quarterly (44:4), pp. iii-vii.

[31] Rawassizadeh, R., Price, B., Petre, M. 2015. "Wearables: Has the Age of Smartwatches Finally Arrived?" Communications of the ACM (58:1), pp. 45-47.

[32] Robson, K., Pitt, L.F., Kietmann, J. 2016. “APC Forum: Extending Business Values through Wearables," MIS Quarterly Executive, (15:2), pp. 167-177.

[33] Schull, N.D. 2016. "Data for Life: Wearable Technology and the Design of Self-Care," BioSocieties (11:3), pp. 317-333. DOI: 10.1057/biosoc.2015.47.

[34] Scott, S.V., Orlikowski, W.J. 2014. "Entanglements in Practice: Performing Anonymity through Social Media," MIS Qarterly (38:3) pp. 873-893.

[35] Spicer, A., Cederstrom, C. 2015. "What Companies Should Ask Before Embracing Wearables", Harvard Business Review (May 20, 2015), pp. 2-5.

[36] Starner, T. 2001. "The Challenges of Wearable Computing: Part 1," IEEE MICRO, pp. 44-52.

[37] Suddaby, R., 2010. "Editor's Comments: Construct Clarity in Theories of Management and Organization," Academy of Management Review (35:3), pp. 346-357.

[38] Swan, M. 2013. "The Quantified Self: Fundamental Disruption in Big Data Science and Biological Discovery," Mary Ann Liebert, Inc. (1:2), pp. 85-99. DOI: 10.1089/big.2012.0002.

[39] Weiser, M. 1991. "The Computer for the $21^{\text {st }}$ Century," Scientific American (265:3), pp. 94-104.

[40] Yoo, Y. 2010. "Computing in everyday life: A call for research on experiential computing," MIS Quarterly (34:2), pp. 213-231. 